\title{
Gender inequality in health among elderly people in a combined framework of socioeconomic position, family characteristics and social support
}

\author{
SILVIA RUEDA* and LUCÍA ARTAZGOZ†
}

\begin{abstract}
This study analyses gender inequalities in health among elderly people in Catalonia (Spain) by adopting a conceptual framework that globally considers three dimensions of health determinants: socio-economic position, family characteristics and social support. Data came from the 2006 Catalonian Health Survey. For the purposes of this study a sub-sample of people aged $65^{-85}$ years with no paid job was selected (I,II3 men and I,484 women). The health outcomes analysed were self-perceived health status, poor mental health status and long-standing limiting illness. Multiple logistic regression models separated by sex were fitted and a hierarchical model was fitted in three steps. Health status among elderly women was poorer than among the men for the three outcomes analysed. Whereas living with disabled people was positively related to the three health outcomes and confidant social support was negatively associated with all of them in both sexes, there were gender differences in other social determinants of health. Our results emphasise the importance of using an integrated approach for the analysis of health inequalities among elderly people, simultaneously considering socio-economic position, family characteristics and social support, as well as different health indicators, in order fully to understand the social determinants of the health status of older men and women.
\end{abstract}

$\boldsymbol{K E Y}$ WORDS - gender, inequalities, elderly, socio-economic factors, family characteristics, social support.

\section{Introduction}

Demographic changes taking place during the last few decades, such as increasing life expectancies and lower fertility rates, have generated population ageing in all parts of the world, but especially in developed

* Universitat Pompeu Fabra, Barcelona, Spain.

† Agència de Salut Pública de Barcelona, and CIBER Epidemiología y Salud Pública (CIBERESP), Spain. 
countries. Between 1960 and 2004, the percentage of those aged up to I4 years old decreased from 25 per cent to I 6 per cent in the 25 European Union countries, whereas the proportion of the population aged 65 and over rose from Io to I2 per cent during the same period and is expected to rise to 30 per cent by 2050. Moreover, the biggest population increase affects those aged over 80 years, the number of whom is expected to double by 2050 to $5 \mathrm{I}$ million citizens (Eurostat 2007). Women account for 59 per cent of the population aged 6o or over in Europe and for 70 per cent of the oldest-old. According to the United Nations' population projections for 2050, Spain will be the second most aged country in the world (after Japan), with 33 per cent of the population 65 or more years and I2 per cent aged 80 and over (United Nations 2006).

These population changes have generated concern around the world about health expenditure and the economic sustainability of the national pension systems. Older people tend to experience more disability, dependency and morbidity, to be more at risk of living alone, and constitute the majority of those with health problems in developed countries (Grundy and Sloggett 2003; IMSERSO 2006a). Little is known, however, about health inequalities in this increasingly important segment of the population, or about the social determinants of their health status, at least as compared with younger people. Most of the studies about social inequalities in health among elderly people conclude that socio-economic inequalities in health prevail in old age (Arber and Ginn I993; Dahl and Birkelund 1997; Marmot and Shipley I996; Rahkonen and Takala ı998; Thorslund and Lundberg 1994). There are, however, still many gaps in our knowledge of social inequalities in health in old age that require further research (Beckett 2000; McMunn et al. 2006; Von Dem Knesebeck et al. 2007).

Research about the social determinants of health among older people has only recently started to integrate three different approaches that were usually studied separately: socio-economic position, family characteristics and social support. Although occupational or social class constitutes one of the most common indicators used in research about social inequalities in health, its measurement among elderly people is controversial because some elderly women have never worked or have had a discontinuous working career because of family duties, especially in southern European countries. Moreover, it has been suggested that social class indicators based on occupation are inadequate for older people because the impact of occupation on health decreases with time since leaving the labour market (Hyde and Jones 2007). Educational qualifications have usually been used instead because they can be applied to all adults and are more stable throughout the life-course (Arber and Cooper 2000; Arber and 
Khlat 2002). In a review of socio-economic indicators in research on health inequalities among elderly people, Grundy and Holt (200I) stated that social class or education combined with a deprivation indicator was the most sensitive indicator.

Whereas health variations among men have traditionally been studied using a social class framework, women have been forgotten or studied through the role approach, emphasising their role in the domestic area (Lahelma et al. 2003; Nathanson i980). Although household composition is considered to be one of the most basic and essential determinants of the well being of older adults (Evandrou et al. 200 ; Zimmer 200I), research on the living arrangements of elderly people has mostly centred on samples made up exclusively of women and assumed their traditional role in family responsibilities, especially in the potential risks among those living alone (Anson I988; Michael et al. 200 ; Sarwari et al. I998). On the other hand, providing direct care to other people has been associated with presenting worse health (Minkler and Fuller-Thompson 200I; Musil and Ahmad 2002), above all among women in relation to stress (Mui I995; Walker, Pratt and Eddy ı995; Pavalko and Woodbury 2000; Hirst 2005). Although informal care to family members has usually referred to women, the literature about care-giving and its impact on health is increasingly incorporating men as important providers of care inside and between households (Baker and Robertson 2008; Crocker 2002; Gregory, Peters and Cameron I990; Horowitz I985; Kaye and Applegate 1993).

Regarding social support, several epidemiological studies have found a positive association with both physical and psychological health among elderly people (Grundy and Sloggett 2003; Oxman et al. I992) and that the association varies by socio-economic position (Oakley and Rajan I99I) and gender (Shye et al. I995). Two types of mechanisms have been described when studying the relationship between social support and health: the direct positive effects of support and the buffering effect, by which social support moderates the impact of acute and chronic stressors on health (Stansfeld I999). Filial obligation in Spain, as in other Mediterranean countries, is a strong value and it has been stated that breaking the intergenerational contract of support has consequences for the physical and mental health of older adults (Zunzunegui et al. 2004).

The aim of this study is to analyse the social determinants of health in the Autonomous Community of Catalonia, Spain using a combined framework of socio-economic position, family roles and social support. The analyses are based on three health indicators shown to be important in gerontological research: self-perceived health, mental health and functional limitations (Beckett et al. i996; Idler and Benyamini i997). 


\section{Methods}

Data

The data are from the 2006 Encuesta Salud de Catalunya (Catalonian Health Survey) (hereafter ESCA 2006), a cross-sectional study that collected information about morbidity, health status, health-related behaviours and use of health care services, as well as socio-demographic data from a representative sample of the non-institutionalised population of Catalonia, a region in the North East of Spain with about seven million inhabitants. In total, I8,I26 subjects were randomly selected using a multiple-stage random sampling strategy with a maximum global error of \pm 0.7 per cent. Trained interviewers administered the questionnaires at people's homes in a face-to-face interviews (Mompart et al. 2007).

For the purposes of this study a sub-sample of people aged $65^{-85}$ years who had no paid job was selected (I,II3 men and I,484 women). The minimum age has been chosen based on the standard legal retirement age in Spain (Consejo Economíco y Social 2000), and the exclusion of all people with paid work is justified by the fact that the meaning of living arrangements and their impact on health depends to a great extent on employment status (Artazcoz et al. 2004). Employment status is not a confounding variable but an interacting variable, i.e. the meaning of family characteristics and socio-economic status can be different and have a different impact on health depending on being in work. Moreover, with the available cross-sectional data it would not be possible to test for the 'healthy worker hypothesis', that good health increases the probability of getting or keeping a paid job (Ross and Mirowsky I995).

The decision to take 85 years as the maximum age, on the other hand, was based on the fact that, although institutionalisation rates in Spain are lower than in other European countries, among those aged 85 and over, they are almost four times higher than among the total elderly population and depend on variables such as sex, socio-economic position, family characteristics and health (Arber and Cooper I999; Grundy and Jitlal 2007; IMSERSO 2006a). More specifically, in Catalonia, the most recent data on institutionalisation rates showed that in January 2006, 75 per cent of elderly residents of public institutions were older than 80 years, and that among them, 83 per cent were women (IMSERSO 2008). Apart from that, taking people younger than 86 reduces the probability of social selection among the oldest old (Idler I993; Orfila et al. 2000; Vuorisalmi, Lintonen and Jylhä 2006). Moreover, those aged over 85 presented a higher non-response rate in some of the predictor variables such as social support ( $37.5 \%$ vs. $5.7 \%$ among $65-85$ years) and in the outcome variable mental health (37.7\% vs. $5.7 \%$ among $65-85$ years). 


\section{Health outcomes}

Self-perceived health status was elicited by asking the respondents to describe their general health as 'excellent', 'very good', 'good' 'fair' or 'poor'. The variable was dichotomised by combining the categories 'fair' and 'poor' to indicate perceived health as below 'good' (Manor, Matthews and Power 2000). Self-perceived health is a broad indicator of health-related wellbeing and has also proved to be a good predictor of mortality (Ferraro and Farmer 1996; Idler and Benyamini 1997; Mossey and Shapiro I982).

Poor mental health status was measured with the I2-item version of the Goldberg General Health Questionnaire (I2-GHQ) (Goldberg et al. I970). This is a screening instrument widely used to detect current, diagnosable psychiatric disorders (Goldberg 1972). The original variable was recoded into a dichotomy, taking scores higher than two to indicate poor mental health status (value I).

Limiting long-standing illness (LLI) was generated through the combination of the questions, 'During the last 12 months have you had any trouble or difficulty for gainful employment, housework, schooling, studying, because of a chronic health problem (that has lasted or it is expected to last three or more months)?' and 'Apart from that considered before, during the last 12 months have you had to restrict or decrease everyday activities such as taking a walk, doing sport, playing, going shopping, etc. because of a chronic health problem?' The final variable was scored 'I' when the interviewee answered positively to at least one of the questions, and ' 0 ' otherwise.

\section{Predictor variables}

Socio-economic position was measured through two indicators: educational attainment and material deprivation. Educational attainment was generated by collapsing some categories of the original variable because of the few individuals in some groups. The final variable was made up of the following categories: more than primary education (reference category), primary education, and less than primary education. Material deprivation was measured through variables measuring household material standards and generated by combining the following five items: having a shower and/or a bath, having hot running water, having central or dispersed heating, having an elevator, and having a washing machine. The resulting variable, household resources, had the following three categories: not lacking any of the items, lacking one of the items and lacking two or more of the items.

Family characteristics were measured through three variables: living arrangements, living with a disabled person in the household and caring 
for a disabled person. Living arrangements were measured through the combination of the variables household size and marital status, generating a four-categories variable to reflect the most usual types of households among the population under study: living with partner (reference category), living alone, not living with partner but living with other people and being the household head, and not living with partner but living with other people and not being the household head. People were asked about living with anyone needing special attention through disability, dependence or limitations in carrying out familiar, social or job-related activities. It had the value ' $\mathrm{I}$ ' when answers were positive, and ' $\mathrm{o}$ ' otherwise. In addition, people were asked about who was the main carer of the disabled person at home. This variable was dichotomised to take the value ' $I$ ' when the respondent stated being the main carer, and 'o' otherwise.

Social support was measured through a reduced version of the original I I-items Duke Social Support Scale, the validity and reliability of which has been demonstrated in several studies in Spain and other countries (Bellón et al. 1996; Broadhead et al. I988; De la Revilla et al. 1991). The version used in ESCA 2006 is based on the first validation of the questionnaire, in which three of the I original items could not be classified into the two dimensions of social support: confidant and affective social support (Broadhead et al. 1988). In the original questionnaire, people where asked eight questions about social support using a Likert-type scale with value ' $I$ ' meaning 'less than desired' and ' 5 ' 'as much as desired'. The Cronbach's alpha coefficients of the two groups of items were 0.87 for the confidant social support questions, and 0.84 for the affective social support ones.

The confidant social support index is the result of combining the responses to the following prompts: 'I get invitations to go out and do things with other people', 'I get chances to talk to someone about problems at work or with my housework', 'I get chances to talk to someone about my personal and family problems', 'I get chances to talk to someone about money matters' and 'I get useful advice about important things in life', and scored from ' 5 ' (minimum confidant social support) to ' 25 ' (maximum confidant social support). The affective social support index is the result of combining the following questions: 'I get love and affection', 'I have people who care what happens to me' and 'I get help when I'm sick in bed', and scored from ' 3 ' (minimum affective social support) to ' 15 ' (maximum affective social support).

\section{Statistical analysis}

Multiple logistic regression models were fitted in order to calculate adjusted odds ratios (aOR) and 95 per cent confidence intervals (CI). 
Separate models were run for each sex. The analysis was carried out following a hierarchical modelling strategy in which the explanatory variables of the conceptual framework were added in three steps (Victoria et al. I997). First, logistic regression models adjusted for age and socio-economic position were fitted (model I). To study the impact of the household characteristics, the type of household and the caring tasks were added at the second step (model 2). Finally, to control by the level of social support, the confidant social support and the affective social support indexes were introduced (model 3). Analyses included weights derived from the complex sample design. Goodness-of-fit was obtained using the Hosmer Lemeshow Test (Hosmer and Lemeshow 2000).

\section{Results}

\section{General description of the population}

Table I profiles the population under study. Women were slightly older than men and had lower educational attainment, whereas levels of material deprivation measured through lack of household resources were similar in both sexes. Regarding type of household, women were more likely than men to live alone $(26 \%$ vs. $9 \%)$ or with people other than the partner both as household head (I0 \% vs. $4 \%$ ) and not as household head ( I $\%$ vs. $3 \%$ ), whilst living with the partner was more frequent among men $(84 \%$ vs. $52 \%)$. Whereas no gender differences were found in living with a disabled person, the percentage of women taking care of disabled people at home was higher than among men (6\% vs. $4 \%)$. Both kinds of social support were high among the men and women in the sample, but especially affective social support. Women were more likely to report poor self-perceived health status, their frequency of poor mental health status was more than double that of men, and they suffered more limiting longterm illnesses (LLI).

\section{Gender differences in health status}

The prevalence of poor health outcomes was significantly higher among women for all three indicators, but especially regarding poor mental health status (Table 2). After adjusting for age and socio-economic position, women were more likely to report poor self-perceived health status $(\mathrm{aOR}=\mathrm{I} .63 ; 95 \% \mathrm{CI}=\mathrm{I} .39-\mathrm{I} .92)$, poor mental health status $(\mathrm{aOR}=$ $2.30 ; 95 \% \mathrm{CI}=\mathrm{I} .78-2.96)$ and LLI (aOR $=\mathrm{I} .78 ; 95 \% \mathrm{CI}=\mathrm{I} .48-2 . \mathrm{I} 4)$. Gender differences in the three health indicators remained after additionally adjusting for household characteristics and social support. 
T A в L E I. General description of the study population (in percentages). Catalonian Health Survey, 2006

\begin{tabular}{|c|c|c|c|}
\hline & $\underset{(n=\mathrm{II} \mathrm{I} 3)}{\operatorname{Men}}$ & $\begin{array}{l}\text { Women } \\
(n=\mathrm{I} 484)\end{array}$ & $p$ \\
\hline Age (median, $25 \%-75 \%$ percentiles) & $73,69-78$ & $74,70-79$ & $<0.001$ \\
\hline $\begin{array}{l}\text { Educational attainment } \\
\text { More than primary schooling } \\
\text { Primary } \\
\text { Less than primary }\end{array}$ & $\begin{array}{l}30.2 \\
33.8 \\
36.0\end{array}$ & $\begin{array}{l}17.8 \\
30.7 \\
5^{\mathrm{I}} \cdot 5\end{array}$ & $<0.001$ \\
\hline $\begin{array}{l}\text { Household resources } \\
\text { o items lacked } \\
\text { I item lacked } \\
2 \text { or more items lacked }\end{array}$ & $\begin{array}{r}63.8 \\
33.5 \\
2.7\end{array}$ & $\begin{array}{r}60.7 \\
37.6 \\
1.7\end{array}$ & 0.302 \\
\hline $\begin{array}{l}\text { Type of household } \\
\text { Living with partner } \\
\text { Living alone } \\
\text { Not living with partner (household head) } \\
\text { Not living with partner (not household head) }\end{array}$ & $\begin{array}{r}84 \cdot 3 \\
8.6 \\
4 \cdot 5 \\
2.6\end{array}$ & $\begin{array}{l}52 . \mathrm{I} \\
25 \cdot 9 \\
\mathrm{I} 0.5 \\
\text { II. } 5\end{array}$ & $0.03^{2}$ \\
\hline $\begin{array}{l}\text { Living with a disabled person } \\
\text { Taking care of a disabled person } \\
\text { Confidant social support }{ }^{1}\end{array}$ & $\begin{array}{l}\text { I } 6.5 \\
3.7 \\
\text { 2I, } 18-24\end{array}$ & $\begin{array}{l}16.4 \\
5.6 \\
20,17-24\end{array}$ & $\begin{array}{l}0.966 \\
0.024 \\
0.001\end{array}$ \\
\hline $\begin{array}{l}\text { Affective social support }{ }^{2} \\
\text { (median, } 25 \%-75 \% \text { percentiles) }\end{array}$ & $\mathrm{I} 4, \mathrm{I} 2-\mathrm{I} 5$ & $\mathrm{I} 4, \mathrm{I} 2-\mathrm{I} 5$ & 0.012 \\
\hline $\begin{array}{l}\text { Self-perceived health } \\
\text { Very good } \\
\text { Good } \\
\text { Fair } \\
\text { Poor } \\
\text { Very poor }\end{array}$ & $\begin{array}{r}3.2 \\
8.8 \\
4^{\mathrm{I}} \cdot 9 \\
3^{6.8} \\
9.4\end{array}$ & $\begin{array}{r}\text { I.I } \\
6.9 \\
30.6 \\
44.5 \\
\text { I6.9 }\end{array}$ & $<0.001$ \\
\hline $\begin{array}{l}\text { Poor mental health status } \\
\text { Limiting long-standing illness }\end{array}$ & $\begin{array}{r}8.9 \\
\text { I9.9 }\end{array}$ & $\begin{array}{l}\mathrm{I} 9 \cdot 9 \\
32.0\end{array}$ & $\begin{array}{l}<0.001 \\
<0.001\end{array}$ \\
\hline
\end{tabular}

1 The Confidant Social Support Index ranges from 5 to 25 .

2 The Affective Social Support Index ranges from 3 to I 5 .

Relationship between the socio-economic position and household characteristics with the health outcomes

Tables 3 to 5 show step-by-step the hierarchical modelling carried out. In Model I, only the socio-economic variables were introduced in the analysis as explanatory variables of the health indicators under study. In both sexes, an association between educational attainment and poor health outcomes was observed and a consistent gradient was found in almost all the health indicators considered. People with less than primary education had the highest probability of reporting a poor self-perceived health status $\left(\mathrm{aOR}=\mathrm{I} .94 ; 95 \% \quad \mathrm{CI}=\mathrm{I} .43^{-2.62}\right.$ among men and 
T A в L E 2. Odds ratios $(a O R)$ and $95 \%$ confidence intervals $(C I)$ comparing health outcomes of women to men. Catalonian Health Survey, 2006

\begin{tabular}{|c|c|}
\hline Health outcome and controls & $\mathrm{aOR}(95 \% \mathrm{CI})$ \\
\hline \multicolumn{2}{|l|}{ Poor self-perceived health status } \\
\hline Adjusted for age & I. $79\left(\mathrm{I} .5^{2-2.09}\right) * * * *$ \\
\hline Adjusted for age and socio-economic position & I.63 (I.39-I.92)*** \\
\hline $\begin{array}{l}\text { Adjusted for age, socio-economic position and } \\
\text { household characteristics }\end{array}$ & $\mathrm{I} .79\left(\mathrm{I} . \mathrm{S}^{\mathrm{I}-2 . \mathrm{I} 2}\right)^{* * * *}$ \\
\hline $\begin{array}{l}\text { Adjusted for age, socio-economic position, household } \\
\text { characteristics and social support }\end{array}$ & $\mathrm{I} .76\left(\mathrm{I} .49^{-2.09}\right)^{* * *}$ \\
\hline \multicolumn{2}{|l|}{ Poor mental health status } \\
\hline Adjusted for age & $2.5 \mathrm{I}(\mathrm{I} .95-3.22)^{* * * *}$ \\
\hline Adjusted for age and socio-economic position & $2.30(\mathrm{r} .78-2.96)^{* * * *}$ \\
\hline $\begin{array}{l}\text { Adjusted for age, socio-economic position and } \\
\text { household characteristics }\end{array}$ & $2.4 \mathrm{I}(\mathrm{I} .86-3 . \mathrm{II})^{* * * *}$ \\
\hline $\begin{array}{l}\text { Adjusted for age, socio-economic position, household } \\
\text { characteristics and social support }\end{array}$ & $2.3^{8}(\mathrm{I} .83-3 . \mathrm{IO}) * * * *$ \\
\hline \multicolumn{2}{|l|}{ Limiting long-standing illness } \\
\hline Adjusted for age & I. $84\left(\mathrm{I} .53^{-2.22}\right) * * * *$ \\
\hline Adjusted for age and socio-economic position & $\mathrm{I} .78\left(\mathrm{I} .4^{8-2.14}\right)^{* * * *}$ \\
\hline $\begin{array}{l}\text { Adjusted for age, socio-economic position and } \\
\text { household characteristics }\end{array}$ & I. $98(\text { I. } 6 \mathrm{I}-2.42)^{* * * *}$ \\
\hline $\begin{array}{l}\text { Adjusted for age, socio-economic position, household } \\
\text { characteristics and social support }\end{array}$ & I. $94\left(\mathrm{I} .5^{8-2.38}\right)^{* * *}$ \\
\hline
\end{tabular}

Significance levels: $* p<0.05 ; * * p<0.01 ; * * *<0.001$.

aOR $=2.55 ; 95 \% \mathrm{CI}=\mathrm{I} .9 \mathrm{I}-3.42$ among women) and a poor-mental health status $(\mathrm{aOR}=\mathrm{I} .83 ; 95 \% \mathrm{CI}=\mathrm{I} .05-3.20$ among men and $\mathrm{aOR}=$ $2.44 ; 95 \% \mathrm{CI}=\mathrm{I} .59-3.75$ among women) compared to those with more than primary education. Low educational attainment was not significantly associated with having a LLI among men, whilst a positive relationship with a gradient was found for women $(\mathrm{aOR}=\mathrm{I} .64 ; 95 \% \mathrm{CI}=\mathrm{I} . \mathrm{I} 8-2.27$ for less than primary education and $\mathrm{aOR}=\mathrm{I} .47 ; 95 \% \mathrm{CI}=\mathrm{I} .04-2.08$ for primary education, compared to more than primary education). Lacking one of the household resources considered in the material deprivation indicator was only positively related to poor mental health status among women $\left(\mathrm{aOR}=\mathrm{I} .5^{\mathrm{I}} ; 95 \% \mathrm{CI}=\mathrm{I} . \mathrm{I} 5^{-\mathrm{I} .98}\right)$, whereas lacking two or more items was only positively related to having a limiting long-standing illness among men $(\mathrm{aOR}=2.19 ; 95 \% \mathrm{CI}=\mathrm{I} .07-4.94)$.

When household characteristics were introduced in Model 2, living alone was the only type of living arrangement significantly associated with health status. Both men and women in this situation were more likely to report poor mental health status as compared to those living with the partner $(\mathrm{aOR}=2.53 ; 95 \% \mathrm{CI}=\mathrm{I} .3 \mathrm{I}-4.89$ and $\mathrm{aOR}=\mathrm{I} .98 ; 95 \% \mathrm{CI}=$ I.39-2.79, respectively), and only among women was it positively 
T А в L E 3. Multivariate associations between poor self-perceived health status and the socio-economic, household living arrangements and social support indicators, men and women 65-85years old, Catalonia 2006

\begin{tabular}{|c|c|c|c|c|}
\hline \multirow{2}{*}{$\begin{array}{l}\text { Gender, attribute } \\
\text { and controls }\end{array}$} & \multicolumn{2}{|r|}{ Model I } & \multirow{2}{*}{$\begin{array}{c}\text { Model } 2 \\
\text { aOR }(95 \% \mathrm{CI})\end{array}$} & \multirow{2}{*}{$\begin{array}{c}\text { Model } 3 \\
\text { aOR }(95 \% \text { CI })\end{array}$} \\
\hline & $\%$ & $\mathrm{aOR}(95 \% \mathrm{CI})$ & & \\
\hline Men & & $n=\mathrm{I} 378$ & $n=\mathrm{I} 299$ & $n=\mathrm{I} 299$ \\
\hline \multicolumn{5}{|l|}{ Educational attainment } \\
\hline More than primary (ref) & 34.9 & I & I & I \\
\hline Primary & $49 \cdot 3$ & $\mathrm{I} .76(\mathrm{I} .3 \mathrm{O}-2.39)^{* * * *}$ & I.90 (I. $38-2.62)^{* * * *}$ & I.89 (I.36-2.6I)**** \\
\hline Less than primary & 52.7 & I. $94\left(\mathrm{I} .43^{-2.62}\right)^{* * *}$ & I.90 (I.38-2.62)**** & I. $83\left(\mathrm{I} .33^{-2.53}\right)^{* * *}$ \\
\hline \multicolumn{5}{|l|}{ Household resources } \\
\hline $\mathrm{o}$ items lacked (ref) & 44.8 & I & I & I \\
\hline I item lacked & 47.7 & $\mathrm{I} .09\left(0.85^{-\mathrm{I} .4 \mathrm{I}}\right)$ & $\mathrm{I} .20(0.9 \mathrm{I}-\mathrm{I} .57$ & I.I $4(0.86-\mathrm{I} .5 \mathrm{O})$ \\
\hline 2 or more items lacked & 60.9 & I. $75(0.82-3.74)$ & I. $74(0.77-3.95)$ & I. $59(0.68-3.67)$ \\
\hline \multicolumn{5}{|l|}{ Type of household } \\
\hline Living with partner (ref) & 46.9 & & I & I \\
\hline Living alone & $4 \mathrm{I} \cdot 4$ & & $0.90\left(0.57^{-1.4 I}\right)$ & $0.80\left(0.5^{-}-\mathrm{I} .29\right)$ \\
\hline $\begin{array}{l}\text { Not living with partner } \\
\text { (household head) }\end{array}$ & 35.0 & & $0.6 \mathrm{I}\left(0.3^{2-1.16)}\right.$ & $0.64\left(0.33^{-1.23)}\right.$ \\
\hline $\begin{array}{l}\text { Not living with partner } \\
\text { (not household head) }\end{array}$ & $5^{8.9}$ & & $\mathrm{I} .27\left(0.5^{0}-3.18\right)$ & I.07 $\left(0.42^{-2.70}\right)$ \\
\hline Living with a disabled person & 63.9 & & 3.IO $(2.06-4.60) * * *$ & $2.85(\mathrm{I} .9 \mathrm{O}-4.28) * * *$ \\
\hline $\begin{array}{l}\text { Taking care of a } \\
\text { disabled person }\end{array}$ & $5^{2.4}$ & & $0.54(0.26-\mathrm{I} . \mathrm{I} 3)$ & $0.5^{2}\left(0.24^{-1.09)}\right.$ \\
\hline Confidant Social Support & - & & & $0.89(0.86-0.94)^{* * *}$ \\
\hline Affective Social Support & - & & & $\mathrm{I} .09(\mathrm{I} .00-\mathrm{I} . \mathrm{I} 9)^{*}$ \\
\hline Women & & $n=\mathrm{I} 734$ & $n=\mathrm{I} 633$ & $n=\mathrm{I} 633$ \\
\hline \multicolumn{5}{|l|}{ Educational attainment } \\
\hline More than primary (ref) & 44.9 & I & I & I \\
\hline Primary & 57.9 & I. $64(\mathrm{I} .2 \mathrm{I}-2.23)^{* * *}$ & I. $66(\mathrm{I} .20-2.28)^{* *}$ & $\mathrm{I} .5^{8}\left(\mathrm{I} . \mathrm{I} 5^{-2.18}\right)^{* *}$ \\
\hline Less than primary & 69.2 & $2.55(\mathrm{I} .9 \mathrm{I}-3.42)^{* * *}$ & $2.48(\text { (. } 83-3.36)^{* * * *}$ & $2.28(\mathrm{I} .68-3 . \mathrm{IO}) * * *$ \\
\hline \multicolumn{5}{|l|}{ Household resources } \\
\hline $\mathrm{o}$ items lacked (ref) & $59 \cdot 4$ & I & I & I \\
\hline I item lacked & 64.5 & I.I2 (0.90-I.4I $)$ & I.05 $(0.83-\mathrm{I} .32)$ & $\mathrm{I} .04(0.82-\mathrm{I} .3 \mathrm{I})$ \\
\hline 2 or more items lacked & 65.5 & I.I5 $\left(0.49^{-2.68}\right)$ & $\mathrm{I} .19\left(0.5^{0}-2.8 \mathrm{I}\right)$ & I.I7 (0.49-2.79) \\
\hline \multicolumn{5}{|l|}{ Type of household } \\
\hline Living with partner (ref) & 62.2 & & I & I \\
\hline Living alone & 57.6 & & $0.93(0.70-1.23)$ & $0.84(0.63-$ I.I 2$)$ \\
\hline $\begin{array}{l}\text { Not living with partner } \\
\text { (household head) }\end{array}$ & 63.0 & & $0.95\left(0.65^{-1.40}\right)$ & $0.9^{2}\left(0.63^{-\mathrm{I}} .37\right)$ \\
\hline $\begin{array}{l}\text { Not living with partner } \\
\text { (not household head) }\end{array}$ & 64.8 & & $0.77\left(0.5^{\mathrm{I}-\mathrm{I} .17}\right)$ & $0.77\left(0.5^{\mathrm{I}-\mathrm{I} .17}\right)$ \\
\hline Living with a disabled person & 78.0 & & $4.46(2.74-7.26)^{* * * *}$ & $4.15\left(2.54^{-6.77}\right)^{* * *}$ \\
\hline $\begin{array}{l}\text { Taking care of a } \\
\text { disabled person }\end{array}$ & 64.9 & & $0.33(0.17-0.64)^{* *}$ & $0.33(0.17-0.64)^{* *}$ \\
\hline Gonfidant Social Support & - & & & $0.93(0.90-0.97)^{* * *}$ \\
\hline Affective Social Support & - & & & I.02 (0.96-I.09) \\
\hline
\end{tabular}

Notes: Adjusted by age. aoR: adjusted odds ratios. CI: 95 per cent confidence interval.

Source: Catalonian Health Survey 2006. For details see text.

Significance levels: $* p<0.05 ; * * p<0.01 ; * * * p<0.001$. 
T А в L Е 4. Multivariate associations between poor mental health status and the socio-economic, household living arrangements and social support indicators, men and women 65-85 years old, Catalonia 2006

\begin{tabular}{|c|c|c|c|c|}
\hline \multirow{2}{*}{$\begin{array}{l}\text { Gender, attribute } \\
\text { and controls }\end{array}$} & \multicolumn{2}{|r|}{ Model I } & \multirow{2}{*}{$\begin{array}{c}\text { Model } 2 \\
\text { aOR }(95 \% \mathrm{CI})\end{array}$} & \multirow{2}{*}{$\begin{array}{c}\text { Model } 3 \\
\text { aOR }(95 \% \mathrm{CI})\end{array}$} \\
\hline & $\%$ & $\mathrm{aOR}(95 \% \mathrm{CI})$ & & \\
\hline Men & & $n=\mathrm{I} 299$ & $n=\mathrm{I} 299$ & $n=\mathrm{I} 299$ \\
\hline \multicolumn{5}{|l|}{ Educational attainment } \\
\hline More than primary (ref) & 6.2 & I & I & I \\
\hline Primary & 8.9 & I. $44(0.80-2.57)$ & I.37 (o.76-2.48) & I.33 (0.73-2.43) \\
\hline Less than primary & II.3 & I. $83(1.05-3.20)^{*}$ & I.74 (0.98-3.07) & I. $46(0.82-2.63)$ \\
\hline \multicolumn{5}{|l|}{ Household resources } \\
\hline o items lacked (ref) & 8.3 & I & I & I \\
\hline I item lacked & $9 \cdot 5$ & I.I3 (0.72-I.77) & I.I7 (0.77-I.86) & I.I2 (0.70-I.8I) \\
\hline 2 or more items lacked & I5.4 & I.89 (0.65-5.52) & $0.74\left(0.22-2.5^{2}\right)$ & $0.85(0.25-2.85)$ \\
\hline \multicolumn{5}{|l|}{ Type of household } \\
\hline Living with partner (ref) & 8.3 & & I & I \\
\hline Living alone & I 4.9 & & $2.53(\mathrm{I} .3 \mathrm{I}-4.89)^{* *}$ & I.49 (o.7I-3.IO) \\
\hline $\begin{array}{l}\text { Not living with partner } \\
\text { (household head) }\end{array}$ & 6.1 & & $0.74\left(0.22-2.5^{2}\right)$ & $0.78(0.23-2.69)$ \\
\hline $\begin{array}{l}\text { Not living with partner } \\
\text { (not household head) }\end{array}$ & $\mathrm{I} 3 \cdot 5$ & & $2.03\left(0.5^{2-7.92)}\right.$ & I.43 (0.35-5.83) \\
\hline Living with a disabled person & I8.4 & & $4.03(2.39-6.79)^{* * * *}$ & $3.69(2.15-6.32)^{* * * *}$ \\
\hline $\begin{array}{l}\text { Taking care of a } \\
\text { disabled person }\end{array}$ & I0.9 & & $0.46(0.15-1.35)$ & $0.38(0.12-\mathrm{I} .20)$ \\
\hline Confidant Social Support & - & & & $0.92(0.86-0.98)^{* *}$ \\
\hline Affective Social Support & - & & & $0.90(0.80-$ I.OI $)$ \\
\hline Women & & $n=\mathrm{I} 633$ & $n=\mathrm{I} 633$ & $n=\mathrm{I} 633$ \\
\hline \multicolumn{5}{|l|}{ Educational attainment } \\
\hline More than primary (ref) & II.I & I & I & I \\
\hline Primary & I7.4 & I. $63($ I.03-2.58)* & I.69 (I.06-2.69)* & I.59 (0.99-2.55) \\
\hline Less than primary & $24 \cdot 7$ & $2.44(\mathrm{I} .59-3.75)^{* * * *}$ & $2.62(\mathrm{I} .69-4.04)^{* * *}$ & $2.39(\mathrm{I} .54-3.73)^{* * *}$ \\
\hline \multicolumn{5}{|l|}{ Household resources } \\
\hline o items lacked (ref) & I6.7 & I & I & I \\
\hline I item lacked & 24.8 & I.5 I (I.I5-I.98)** & I.4I (I.07-I.86)* & I.39 (I.05-I.85)* \\
\hline 2 or more items lacked & 27.1 & I.69 (o.68-4.19) & I. $65(0.66-4 . \mathrm{I} 3)$ & I.5I (o.59-3.89) \\
\hline \multicolumn{5}{|l|}{ Type of household } \\
\hline Living with partner (ref) & $\mathrm{I} 7 \cdot 4$ & & I & I \\
\hline Living alone & 23.7 & & I.98 (I.39-2.79)**** & I.60 (I.II-2.29)* \\
\hline $\begin{array}{l}\text { Not living with partner } \\
\text { (household head) }\end{array}$ & 22.3 & & I.3I $\left(0.83^{-2.06}\right)$ & $\mathrm{I} .23\left(0.77^{-1} .94\right)$ \\
\hline $\begin{array}{l}\text { Not living with partner } \\
\text { (not household head) }\end{array}$ & 20.9 & & $\mathrm{I} .35(0.82-2.23)$ & $\mathrm{I} .45(0.87-2.42)$ \\
\hline Living with a disabled person & $29 \cdot 5$ & & $2.72(\mathrm{I} .8 \mathrm{I}-4.09) * * *$ & $2.49($ I.64-3.79)*** \\
\hline $\begin{array}{l}\text { Taking care of a } \\
\text { disabled person }\end{array}$ & 22.6 & & $0.60\left(0.3^{2-I . I 3}\right)$ & $0.59(0.3 \mathrm{I}-\mathrm{I} .24)$ \\
\hline Confidant Social Support & - & & & $0.95(0.9 \mathrm{I}-0.99)^{*}$ \\
\hline Affective Social Support & - & & & $0.89(0.83-0.96)^{* *}$ \\
\hline
\end{tabular}

Notes: Adjusted by age. aoR: adjusted odds ratios. CI: 95 per cent confidence interval.

Source: Catalonian Health Survey 2006. For details see text.

Significance levels: $* p<0.05 ; * * p<0.01 ; * * * p<0.001$. 
T А в L E 5. Multivariate associations between limiting long-standing illness and the socio-economic, household living arrangements and social support indicators, men and women 65-85 years old, Catalonia 2006

\begin{tabular}{|c|c|c|c|c|}
\hline \multirow{2}{*}{$\begin{array}{l}\text { Gender, attribute } \\
\text { and controls }\end{array}$} & \multicolumn{2}{|r|}{ Model I } & \multirow{2}{*}{$\begin{array}{c}\text { Model } 2 \\
\operatorname{aOR}(95 \% \mathrm{CI})\end{array}$} & \multirow{2}{*}{$\begin{array}{c}\text { Model } 3 \\
\operatorname{aOR}(95 \% \mathrm{CI})\end{array}$} \\
\hline & $\%$ & $\mathrm{aOR}(95 \% \mathrm{CI})$ & & \\
\hline Men & & $n=\mathrm{I} 378$ & $n=\mathrm{I} 299$ & $n=\mathrm{I} 299$ \\
\hline \multicolumn{5}{|l|}{ Educational attainment } \\
\hline More than primary (ref) & 20.2 & I & I & I \\
\hline Primary & I8.4 & $0.88(0.60-\mathrm{I} .28)$ & $0.9 \mathrm{I}\left(0.6 \mathrm{I}-\mathrm{I} .3^{8}\right)$ & $0.96(0.64-\mathrm{I} .45)$ \\
\hline Less than primary & $2 \mathrm{I} .2$ & $\mathrm{I} .04(0.72-\mathrm{I} .50)$ & $0.9^{8}\left(0.65^{-\mathrm{I} .46)}\right.$ & $0.90\left(0.59^{-\mathrm{I}} .35\right)$ \\
\hline \multicolumn{5}{|l|}{ Household resources } \\
\hline o items lacked (ref) & 20.4 & I & I & I \\
\hline I item lacked & 17.7 & $0.83(0.60-\mathrm{I} .15)$ & $0.96(0.67-\mathrm{I} .37)$ & $0.93\left(0.65^{-\mathrm{I}} .3^{2}\right)$ \\
\hline 2 or more items lacked & $37 \cdot 7$ & $2.19(1.07-4.94)^{*}$ & $2.62(\mathrm{I} . \mathrm{I} 4-6.02)^{*}$ & $2.5^{\mathrm{I}}(\mathrm{I} .08-5.86)^{*}$ \\
\hline \multicolumn{5}{|l|}{ Type of household } \\
\hline Living with partner (ref) & I9.6 & & I & I \\
\hline Living alone & I9.0 & & I.39 (0.78-2.47) & $\mathrm{I} .37(0.76-2.50)$ \\
\hline $\begin{array}{l}\text { Not living with partner } \\
\text { (household head) }\end{array}$ & 20.7 & & I.I3 $\left(0.53^{-2.44}\right)$ & I.I7 $\left(0.54^{-2.53)}\right.$ \\
\hline $\begin{array}{l}\text { Not living with partner } \\
\text { (not household head) }\end{array}$ & $33 \cdot 3$ & & I.5I $(0.49-4.7 \mathrm{I})$ & I.42 (0.45-4.54) \\
\hline Living with a disabled person & 37.2 & & $4.5^{2}(3.0 \mathrm{I}-6.80)^{* * *}$ & $4.33(2.87-6.53)^{* * *}$ \\
\hline $\begin{array}{l}\text { Taking care of a } \\
\text { disabled person }\end{array}$ & $23 \cdot I$ & & $0.3^{8}(\mathrm{o} . \mathrm{I} \mathrm{I}-0.86)^{*}$ & $0.39(0.16-0.84)^{*}$ \\
\hline Confidant Social Support & - & & & $0.95(0.89-0.99)^{*}$ \\
\hline Affective Social Support & - & & & I.07 (0.96-I.I8) \\
\hline Women & & $n=\mathrm{I} 734$ & $n=\mathrm{I} 633$ & $n=\mathrm{I} 633$ \\
\hline \multicolumn{5}{|l|}{ Educational attainment } \\
\hline More than primary (ref) & $23 \cdot 5$ & I & I & I \\
\hline Primary & $3^{1.7}$ & I.47 (I.04-2.08)* & I. $3^{8}\left(0.9^{6-I} .9^{8}\right)$ & I.3I (o.9I-I.89) \\
\hline Less than primary & $35 \cdot 0$ & I. $64($ I.18-2.27)** & $\mathrm{I} .57(\mathrm{I} . \mathrm{II}-2.2 \mathrm{O})^{*}$ & $\mathrm{I} .42(\mathrm{I} . \mathrm{OI}-2 . \mathrm{OI})^{*}$ \\
\hline \multicolumn{5}{|l|}{ Household resources } \\
\hline o items lacked (ref) & 30.5 & I & I & I \\
\hline I item lacked & $34 \cdot 7$ & I.I6 (0.92-I.45) & I.I3 (0.88-I.45) & I.II (o.87-I.43) \\
\hline 2 or more items lacked & 26.6 & $0.77\left(0.3^{1-1} .88\right)$ & $0.83\left(0.33^{-2.08}\right)$ & $0.77\left(0.30^{-}-\mathrm{I} .97\right)$ \\
\hline \multicolumn{5}{|l|}{ Type of household } \\
\hline Living with partner (ref) & $29 \cdot 7$ & & I & I \\
\hline Living alone & $3^{\mathrm{I}} \cdot 5$ & & I.39 (I.02-I.88)* & I.20 (0.88-I.64) \\
\hline $\begin{array}{l}\text { Not living with partner } \\
\text { (household head) }\end{array}$ & 37.2 & & $1.26\left(0.85^{-1.87)}\right.$ & $\mathrm{I} .2 \mathrm{I}(0.8 \mathrm{I}-\mathrm{I} .79)$ \\
\hline $\begin{array}{l}\text { Not living with partner } \\
\text { (not household head) }\end{array}$ & $3^{8.6}$ & & I.02 (0.66-I.6o) & $\mathrm{I.05}(0.67-\mathrm{I} .65)$ \\
\hline Living with a disabled person & $4^{6.4}$ & & $3.45(2.39-4.98)^{* * *}$ & $3.20(2.20-4.64)^{* * *}$ \\
\hline $\begin{array}{l}\text { Taking care of a } \\
\text { disabled person }\end{array}$ & 33.2 & & $0.44\left(0.25^{-0.77}\right)^{* *}$ & $0.43(0.24-0.76)^{* *}$ \\
\hline Confidant Social Support & - & & & $0.94(0.9 \mathrm{I}-0.98)^{* *}$ \\
\hline Affective Social Support & - & & & $0.97(0.90-1.03)$ \\
\hline
\end{tabular}

Notes: Adjusted by age. aoR: adjusted odds ratios. CI: 95 per cent confidence interval.

Source: Catalonian Health Survey 2006. For details see text.

Significance levels: $* p<0.05 ; * * p<0.01 ; * * * p<0.001$. 
associated with LLI (aOR = I.39; 95\% CI = I.02- $\mathrm{I} .88)$. When controlling for social support in Model 3 , however, living alone was only significantly associated with poor mental health among women. Living with a disabled person was positively and strongly associated with all the health indicators in both sexes, even after adding social support in the analysis. Taking care of disabled people at home, however, was negatively associated with having a LLI in both sexes $(\mathrm{aOR}=0.38 ; 95 \% \mathrm{CI}=0 . \mathrm{I} \mathrm{I}-\mathrm{O} .86$ among men and $\mathrm{aOR}=0.44 ; 95 \% \mathrm{CI}=0.25^{-0.77}$ among women) and with having a poor self-perceived health status among women $(\mathrm{aOR}=0.33 ; 95 \%$ $\mathrm{CI}=0 . \mathrm{I} 7-0.64)$.

In Model 3, subjective social support, disaggregated in confidant and affective social support, was introduced together with all the other explanatory variables of the study. Confidant social support was negatively associated with all the health indicators in both sexes, whilst affective social support was only negatively and significantly associated with poor mental health status among women $(\mathrm{aOR}=0.89 ; 95 \% \mathrm{CI}=0.83-0.96)$ and positively associated with poor self-perceived health status among men $(\mathrm{aOR}=\mathrm{I} .09 ; 95 \% \mathrm{CI}=\mathrm{I} .00-\mathrm{I} . \mathrm{I} 9)$.

\section{Discussion}

This study is a contribution to the relatively new but growing literature about the multiple determinants of health inequalities among older people. As in Grundy and Sloggett's study (2003) carried out in England, we have included different dimensions of health status and of its determinants. Regarding health indicators, however, we have included one closely related to the age group under study, that is, long-standing illnesses generating functional limitations. And regarding the predictor variables, our study overcomes some shortcomings of previous research and provides other important dimensions that are not usually considered. First of all, educational attainment had three categories instead of being a dichotomous variable, making it possible to analyse the socio-economic gradient in health inequalities. Moreover, household living arrangements was used instead of marital status, a much more important determinant of wellbeing among elderly people, together with two other dimensions of household characteristics: living with a disabled person and taking care of a disabled person. Finally, social support has been measured with two dimensions, showing that the relationship between each of them and health is different depending on the kind of social support received.

The main findings of the study can be summarised as follows. First, as is also the case in younger adults, health status among elderly women is 
poorer than among the men in the three dimensions of health considered. Secondly, even after controlling for social support, living with a disabled person is positively related to all the health indicators considered and in both sexes, whereas taking care of disabled people at home is negatively associated with having a LLI in both sexes and with having a poor selfperceived health among women. Thirdly, whereas living alone was associated with poor mental health status in both sexes, the association disappeared among men after adjusting for social support. Finally, confidant social support is negatively related to poor health status, whereas affective social support only behaves this way with poor mental health among women.

\section{Gender differences in health status}

The results show that elderly Catalonian women have a poorer selfperceived health status, a poorer mental health status and are more likely to report LLI than their male counterparts. Gender inequalities in health among older people are especially important regarding poor mental health, with women presenting a probability of suffering from it almost two-and-a-half times higher than men. These differences remained after controlling for all the other variables. The higher prevalence of mental health problems among women in all age groups has been reported in other studies (Sonnenberg et al. 2000; Zunzunegui et al. 1998). The different gender patterns depending on the health indicator analysed, as well as the differences in factors associated with each of them point out the importance of examining different health indicators in trying to understand fully the complexity of inequalities in health (Lahelma et al. I999; Macintyre et al. 1996; Matthews, Manor and Power 1999).

Relationship of the socio-economic position, household characteristics and social support with the health outcomes by sex

Some research about social inequalities in health among elderly people has suggested using a set of measures of socio-economic position instead of a single indicator in order to explore the multidimensional nature socioeconomic position has in old age (Avlund et al. 2003; Dalstra et al. 2006; Grundy and Holt 200i; Huisman, Kunst and Mackenbach 2003; Von Dem Knesebeck et al. 2007). Accordingly, two different indicators were used in our study. Educational attainment was more related to the health of women and especially to self-perceived health status, in line with the claim that educational level is a better indicator of health inequalities for women (Arber and Khlat 2002). The socio-economic gradient in health 
among elderly people according to educational attainment found in the present study is consistent with previous research (Dalstra et al. 2006; Huisman, Kunst and Mackenbach 2003).

Material deprivation, as a measure of household material standards of living, was only related to poor mental health among women and more strongly to having a LLI among men after controlling for all the other variables. This result contrasts with other studies in which measures of material deprivation were more strongly associated with poor health among women than men (Borrell et al. 2004; Grundy and Sloggett 2003), but is in line with the finding of an association between material deprivation and poor mental health (Eachus et al. 1996; Groffen et al. 2007).

Anson (I988) found that women living with a partner were the healthiest and women living alone or being head of families were the least healthy, which pointed to the importance of adult support for health status. Consistently, living alone was associated with poor mental health in both sexes and with having a LLI among women, although only the association between living alone and poor mental health among women persisted (albeit weakened) after controlling for social support. This result is in line with those of a study carried out among 6o-72 year-old nurses which found that social engagement and social network variables were associated with a decreased risk of decline in mental health among women living alone (Michael et al. 200i).

Our findings suggest that living alone can have different meanings for elderly men and women, with a high negative impact on women's mental health. A possible explanation of this outcome is the phenomenon of the 'feminisation of poverty' (Pearce I978), together with higher widowhood rates among women, which especially applies in Spain, where many elderly widows live with very small pensions. The association between deprivation and poor mental health among women would support this hypothesis. As also found by the Caregiver Health Effects Study (Schulz and Beach I999) in a sample of Americans aged 66 to 96 years, living with a disabled person was positively and strongly related to poor health; but unlike that study, we found that caring for a disabled person was negatively related to poor health. Surprisingly, whereas taking care of a disabled person presented a negative association with having a poor selfperceived health among women and with having a LLI in both sexes, living with a disabled person was positively and strongly related to all the health outcomes among both men and women, even after controlling for social support. These findings could be explained by a probable reverse causation effect, whereby those taking care of a disabled person would represent a selection of the healthiest elderly, whereas living with a disabled 
and not taking care of him or her could be related to a higher prevalence of poor health status. Shulz and Beach (1999), for instance, found that individuals with a disabled spouse who were not providing care had higher rates of prevalent disease compared to the other three caregiving groups analysed.

Confidant social support was negatively associated with having a poor self-perceived health status, poor mental health and a LLI, whilst affective social support was only negatively related to poor mental health among women and positively associated with poor self-perceived health status among men. Perceived support has been found to protect individuals from the effects of stress (Cohen and Wills 1985, Kessler and McLeod 1985, Wethington and Kessler 1986) and to attenuate the effect of disability on depressive symptoms (Allen, Ciambrone and Welch 2000; Jang et al. 2002; Taylor and Lynch 2004; Turner and Noh I988). In a study carried out in Spain, it has been found that those elderly people with more social links presented lower risks of mortality, cognitive deterioration, depression and disability, and even higher probabilities of recovering after a disability (Otero et al. 2006). This study, however, shows that affective social support is positively related to poor self-perceived health status among men. A possible explanation of this outcome is that elderly men with poor selfperceived health receive more attention from their spouses or other family members. This, however, is a speculation that deserves further investigation.

Although family networks are an important source of support in Spain, the family has been found to be more likely to provide both positive and negative interactions than friends (Aneshensel, Pearlin and Schuler 1993; Antonucci I990; Rogers 1996). Some studies describe the existence of a hierarchical order in the effect of the provision of support on depressive symptoms among elderly people, emotional support from friends (more likely to provide confidant social support) being more important than that from the family (more likely to provide affective social support) (Dean, Kolody and Wood I990; Harlow, Goldberg and Comstock r99i). In line with this evidence, in this study both affective and confidant social support protect elderly women against poor mental health, whereas in the case of men only confidant social support is significantly and negatively related to poor mental health. Given the nature of the sample, however, we cannot rule out the possibility that those with poor mental health receive the least support - the so-called 'contamination hypothesis' (George et al. I989). Previous research, however, has demonstrated less support for the hypothesis of mental health affecting perceived social support than for perceived social support affecting mental health (Taylor and Lynch 2004). 


\section{Limitations}

One of the limitations of this study is its cross-sectional design, a fact that prevents us from determining the directions of causation. For example, as mentioned before, the relationships between living arrangements, caring activities and social support with health are likely to be reciprocal. However, some possible explanations for both causality directions have been provided.

A second limitation seems from the nature of the sample. Limiting the study to community-residing people may have biased the results in the sense that, as men are more likely to have a spouse caring for them when disabilities appear in old age, women have a higher probability of being excluded from the sample because of their higher institutionalisation rates (Marmot, Koveginas and Elston 1987; United Nations 2005). It would be expected that less healthy women would be excluded from the study but yet there was still an excess of female morbidity for the three health indicators analysed. Institutionalisation rates in Spain, however, are among the lowest in Europe (IMSERSO 2006a).

Moreover, the way the variable dealing with taking care of a disabled person has been generated could explain the unexpected outcome, whereby those doing so were healthier. In this study, carers were considered as those defining themselves as the main carers of the disabled persons at home. Perhaps the model could be improved by taking into account the amount of care provided, but unfortunately this was not possible with the original database.

\section{Policy implications}

This study has provided evidence of the importance of simultaneously considering socio-economic position, household characteristics and social support, as well as different health outcomes, in order fully to understand health inequalities among elderly people. It has also emphasised the importance of examining family roles and health not only among women but also among men, as well as the different effects that gender patterns in old age have on different dimensions of health. An integrated approach to socio-economic inequalities, simultaneously studying indicators of household living standards, household structure and social support is needed both in research on inequalities in health as well as in social and health policies addressed to elderly people. Moreover, this study sheds some light on the mechanisms explaining gender inequalities in health among elderly people in Mediterranean countries. Unlike previous research, the hierarchical modelling strategy followed here enabled us to see the impact on health of the three dimensions examined by adding them 
step-by-step, that is, socio-economic position, family characteristics and social support.

In Spain, as in the rest of Europe, the majority of elderly people prefer to live in their homes $(77 \%)$, and only with their children or in institutions as the last options in case of need (IMSERSO 2007). On November 3oth 2006, the Act for the Promotion of Personal Autonomy and Care for Dependent Persons was passed in the Congress of Deputies, with implementation commencing at the end of 2007 and constituting a step forward in social policy in Spain (IMSERSO 2006b). The results of this study show the importance of developing specific policies oriented towards elderly people facing disabilities and their families, such as the one mentioned above. 'Ageing at home' requires the expansion of public care services, to date very underdeveloped in Spain, such as respite services to the family of the dependants, the expansion of home visits to elderly people by health professionals, and the adaptation of housing to the ageng process (e.g. installing elevators in flats and showers instead of baths).

\section{Acknowledgments}

The study was partially financed by Pompeu Fabra University (Universitat Pompeu Fabra) and the Barcelona Public Health Agency (Agència de Salut Pública de Barcelona).

\section{References}

Allen, S. M., Ciambrone, D. and Welch, L. C. 200o. Stage of life course and social support as a mediator of mood stage among persons with disability. Fournal of Aging and Health, $\mathbf{1} 2$, 3, 318-4I.

Aneshensel, C. S., Pearlin, L. I. and Schuler, R. H. I993. Stress, role captivity, and the cessation of caregiving. Fournal of Health and Social Behavior, 34, I, 54-70.

Anson, O. 1988. Living arrangements and women's health. Social Science and Medicine, 26, 2, $20 \mathrm{I}-8$.

Antonucci, T. C. I99o. Social supports and social relationships. In Binstock, R. H and George, L. K. (eds), Handbook of Aging and the Social Sciences. 3rd edition, Academic, New York, 205-6.

Arber, S. and Cooper, H. I999. Gender differences in health in later life: the new paradox? Social Science and Medicine, 48, I, 6I-76.

Arber, S. and Cooper, H. 200o. Gender and inequalities in health across the life course. In Annandale, E and Hunt, K. (eds), Gender Inequalities in Health. Open University Press, Buckingham, UK, I23-49.

Arber, S. and Ginn, J. I993. Gender and inequalities in health in later life. Social Science and Medicine, 36, I, 33-46.

Arber, S. and Khlat, M. 2002. Introduction to social and economic patterning of women's health in a changing world. Social Science and Medicine, 54, 5, 643-47. 
Artazcoz, L., Borrell, C., Benach, J., Cortès, I. and Rohlfs, I. 2004. Women, family demands and health: the importance of employment status and socio-economic position. Social Science and Medicine, 59, 2, 263-74.

Avlund, K., Holstein, B. E., Osler, M., Damsgaard, M. Y., Holm-Pedersen, P. and Rasmussen, N. K. 2003. Social position and health in old age: The relevance of different indicators ofsocial position. Scandinavian Fournal of Public Health, 31, 2, I26-36.

Backer, K. and Robertson, N. 2008. Coping with caring for someone with dementia: reviewing the literature about men. Aging and Mental Health, I 2, 4, 4I3-22.

Beckett, L., Brock, D., Lemke, J., Mendes de Leon, C., Guralnik, J., Fillenbaum, G., Branch, L., Wetle, T. and Evans, D. I996. Analyses of change in self-reported physical functioning among older persons in four population studies. American Fournal of Epidemiology, $\mathbf{1 4 3}, 8,766-78$.

Beckett, M. 2000. Converging health inequalities in later life: an artifact of mortality selection? Journal of Health and Social Behavior, 4I, I, I6-ig.

Bellón, J. A., Delgado, A., de Dios, J. and Lardelli, P. i996. Validez y fiabilidad del cuestionario de apoyo social funcional. DUKE-UNG-I I [Validity and reliability of the functional social support questionnaire, DUKE-UNC-II]. Atención Primaria, I8, 4I53-63.

Borrell, C., Muntaner, C., Benach, J. and Artazcoz, L. 2004. Social class and selfreported health status among men and women: what is the role of work organisation, household material standards and household labour? Social Science and Medicine, 58, io, I869-87.

Broadhead, W. E., Gehlbach, S. H., Degruy, F. V. and Kaplan, B. H. i988. The DukeUNG functional social support questionnaire: measurement of social support in family medicine patients. Medical Care, 26, 7, 709-23.

Consejo Economíco y Social (CES) 200o. Vida laboral y prejubilaciones [Working Life and Preretirement]. Report 2/2002, CES, Madrid.

Cohen, S. and Wills, T. A. I985. Stress, social support, and the buffering hypothesis. Psychological Bulletin, 98, 310-57.

Crocker Houde, S. 2002. Methodological issues in male caregiver research: an integrative review of the literature. Fournal of Advanced Nursing, 4o, 6, 626-40.

Dahl, E. and Birkelund, E. I997. Health inequalities in later life in a social democratic welfare state. Social Science and Medicine, 44, 6, 87 I-8I.

Dalstra, J. A. A., Kunst, A. E., Mackenbach, J. P. and the EU Working Group on Socioeconomic Inequalities in Health 2006. A comparative appraisal of the relationship of education, income and housing tenure with less than good health among elderly people in Europe. Social Science and Medicine, 62, 8, 2046-6o.

De la Revilla, L., Bailón, E., De Dios, J., Delgado, A., Prados, M. A. and Fleitas, L. I99i. Validación de una escala de apoyo social funcional para su uso en la consulta del médico de familia [Validity of a functional social suport scale for its use on the primary health care]. Atención Primaria, 8, 688-92.

Dean, A., Kolody, B. and Wood, P. I99o. Effects of social support from various sources on depression in elderly persons. Fournal of Health and Social Behavior, 31, 2, I48-6I.

Eachus, J., Williams, M., Chan, P., Davey Smith, G., Grainge, M., Donovan, J. and Frankel, S. 1996. Deprivation and cause-specific morbidity: evidence from the Somerset and Avon Survey of Health. British Medical Journal, 31 2, 287-92.

Eurostat. 2007. Europe in Figures. Eurostat Yearbook 2006-07. Office for Official Publication of the European Communities, Luxembourg.

Evandrou, M., Falkingham, J., Rake, K., Scott, A. and The Research Group Simulating Social Policy for an Ageing Society (SAGE) 2001. The dynamics of living arrangements in later life: evidence from the British Household Panel Survey. Population Trends, ro5, I, $37-44$. 
Ferraro, K. and Famer, M. 1996. Double jeopardy. Aging as leveler, or persistant health inequality? A longitudinal analysis of white and black Americans. Fournal of Gerontology, 5I, 6, S319-28.

George, L. K., Blazer, D. G., Hughes, D. C. and Fowler, N. I989. Social support and the outcome of major depression. British Fournal of Psychiatry, I 54, 478-85.

Goldberg, D. 1972. The Detection of Psychiatric Illness by Questionnaire. Oxford University Press, Oxford.

Goldberg, D., Cooper, B., Eastwood, M. R., Kedwart, H. B. and Shepherd M. I970. A standardised psychiatric interview for use in community surveys. British Fournal of Preventive Social Medicine, 24, I, I8-23.

Groffen, D., Bosma, H., Van den Akker, M., Kempen, G. and van Eijk, J. 2007. Material deprivation and health-related dysfunction on older Dutch people: findings from the SMILE study. European Fournal of Public Health, I8, 3, 258-63.

Gregory, D. M., Peters, N. and Cameron, C. F. I990. Elderly male spouses as caregivers: toward an understanding of their experience. Fournal of Gerontological Nursing, r 6, I, 20-4.

Grundy, E. and Holt, G. 200I. The socioeconomic status of older adults: how should we measure it in studies of health inequalities? Journal of Epidemiology and Community Health, 55, I2, 895-904.

Grundy, E. and Jitlal, M. 2007. Socio-demographic variations in moves to institutional care 1991-200I: a record linkage study from England and Wales. Age and Ageing, 36, 4, 424-30.

Grundy, E. and Sloggett, A. 2003. Health inequalities in the older population: the role of personal capital, social resources and socio-economic circumstances. Social Science and Medicine, 56, 5, 935-47.

Harlow, S. D., Goldberg, E. L. and Comstock, G. W. ı99ı. A longitudinal study of risk factors for depressive symptomatology in elderly widowed and married women. American Journal of Epidemiology, $\mathbf{1 3 4}, 5,526-38$.

Hirst, M. 2005. Carer distress: a prospective, population-based study. Social Science and Medicine, 6 $\mathbf{1}, 3,697-708$.

Horowitz, A. I985. Sons and daughters as caregivers to older parents: difference in role performance and consequences. The Gerontologist, 25, 6, 6r2-17.

Hosmer, D. and Lemeshow, S. 2000. Applied Logistic Regression. Second edition, Wiley, New York.

Huisman, M., Kunst, A. E. and Mackenbach, J. P. 2003. Socioeconomic inequalities in morbidity among the elderly: a European overview. Social Science and Medicine, 57, 5, $86 \mathrm{I}-73$.

Hyde, M. and Jones, I. R. 2007. The long shadow of work? Does time since labour market exit affect the association between socio-economic position and health in a post-working population? Fournal of Epidemiology and Community Health, 6 $\mathbf{1}, 6,53^{2-} 3^{8}$.

Idler, E. L. I993. Age differences in self-assessments of health: age changes, cohort differences, or survivorship? Fournal of Gerontology, 48, 6, $\mathrm{S}_{289}-300$.

Idler, E. L. and Benyamini, Y. 1997. Self-rated health and mortality: a review of twentyseven community studies. Fournal of Health and Social Behavior, 38, I, 2 I-37.

IMSERSO 2006 a. Las personas mayores en España. Informe 2006 [Older People in Spain. 2006 Report]. Volume I, Institute for Older Persons and Social Services, Ministry of Labour and Social Affairs, Madrid.

IMSERSO 2006 b. Ley de Promoción de la Autonomía Personal y Atención a las personas en situación de dependencia [Law on the Promotion of Personal Autonomy and Care for Dependent Persons]. Institute of the Elderly and Social Services, Ministry of Labour and Social Affairs, Madrid.

IMSERSO 2007. A Propósito de las condiciones de vida de las personas mayores. Encuesta 2006. [About Elderly People's Life Conditions. 2006 Survey]. Observatory for Elderly People, Ministry of Labour and Social Affairs, Madrid. 
IMSERSO 2008. Las personas mayores: Indicadores claves por Comunidades Autónomas [Elderly People: Key Indicators by Autonomous Communities]. Available online at http://www.segsocial.es/imserso/estadisticas/I_estadisticas.html [Accessed March 2008].

Jang, Y., Haley, W. E., Small, B. J. and Mortimer, J. A. 2002. The role of mastery and social resources in the associations between disability and depression in later life. The Gerontologist, $\mathbf{4 2}^{2}, 6$, 807-13.

Kaye, L. W. and Applegate, J. S. I993. Family support groups for male caregivers: benefits of participation. Fournal of Gerontological Social Work, 2o, 3/4, I67-85.

Kessler, R. C. and McLeod, J. D. I985. Social support and mental health in community samples. In Cohen, S and Syme, S. L. (eds), Social Support and Health. Academic Orlando, Florida, 219-40.

Lahelma, E., Arber, S., Kivelä, K. and Roos, E. 2003. Multiple roles and health among British and Finnish women: the influence of socio-economic circumstances. In Arber, S and Khlat, M. (eds), Social and Economic Patterning of Health among Women. Committee for International Research in Demography, Paris, I75-202.

Lahelma, E., Martikainen, P., Rahkonen, O. and Silventoinen, K. I999. Gender differences in ill-health in Finland: patterns, magnitude and change. Social Science and Medicine, 48, I, 7-I9.

Macintyre, S., Kunt, H. and Sweeting, H. I996. Gender differences in health: are things really as simple as they seem? Social Science and Medicine, 42, 4, 6r 7-24.

Manor, O., Matthews, S. and Power, C. 2000. Dichotomous or categorical response? Analysing self-rated health and lifetime social class. International Fournal of Epidemiology, 29, I, I49-57.

Marmot, M., Kogevinas, M. and Elston, M. 1987. Social/economic status and disease. Annual Review of Public Health, 8, in I-35.

Marmot, M. and Shipley, M. I996. Do socio-economic differences in mortality persist after retirement? 25 years follow up of civil servants from the first Whitehall Study. British Medical Fournal, 31 3, гі77-80.

Matthews, S., Manor, O. and Power, C. I999. Social inequalities in health: are there gender differences? Social Science and Medicine, 48, I, 49-6o.

McMunn, A., Breeze, E., Goodman, A., Nazroo, J. and Oldfield, Z. 20o6. Social determinants of health in older age. In Marmot, M and Wilkinson, R. G. (eds), Social Determinants of Health. Oxford University Press, Oxford, 267-96.

Michael, Y., Berkman, L., Colditz, G. and Kawachi, I. 200I. Living arrangements, social integration, and change in functional health status. American Fournal of Epidemiology, I53, 2, I23-3I.

Minkler, M. and Fuller-Thomson, D. 200I. Physical and mental health status of American grandparents providing extensive care to their children. Fournal of American Medicine Women's Association, 56, 4, I99-205.

Mompart, A., Medina, A., Brugulat, P. and Tresserras, R. 2007. Encuesta de Salud de Catalunya 2006. Un instrumento de planificación sanitaria. [Catalonian Health Survey 2006. A tool for health planning]. Índice. Revista de Estadística y Sociedad, 2o, 19-22.

Mossey, J. and Shapiro, E. I982. Self-rated health: a predictor of mortality among the elderly. American Fournal of Public Health, 72, 8, 80o-8.

Mui, A. I995. Caring for frail elderly parents: a comparison of adult sons and daughters. The Gerontologist, 35, I, 86-93.

Musil, C. and Ahmad, M. 2002. Health of grandmothers: a comparison by caregiver status. Fournal of Aging and Health, I4, I, 96-I2 I.

Nathanson, C. A. I980. Social roles and health status among women: the significance of employment. Social Science and Medicine, I 4a, 6, 463-71.

Oakley, A. and Rajan, L. I99I. Social class and social support, the same or different? Sociology, 25, I, 3 ${ }^{-}-59$. 
Orfila, F., Ferrer, M., Lamarca, R. and Alonso, J. 200o. Evolution of self-rated health status in the elderly: cross-sectional vs. longitudinal estimates. Fournal of Clinical Epidemiology, 53, 6, 563-570.

Otero, A., Zunzunegui, M. V., Béland, F., Rodríguez Laso, A. and García de Yebenes, M. J. 2006. Relaciones sociales y envejecimiento saludable [Social Relations and Healthy Ageing. Working Paper 9] Fundación BBVA, Madrid.

Oxman, T. E., Berkman, L. F., Kasl, S., Freeman, D. H. and Barret, J. I992. Social support and depressive symptoms in the elderly. American Fournal of Epidemiology, $\mathbf{1 3 5}, 4,356-68$.

Pavalko, E. and Woodbury, S. 2000. Social roles as process: caregiving careers and women's health. Fournal of Health and Social Behavior, 41, I, 91-I05.

Pearce, D. 1978. The feminisation of poverty: women, work and welfare. Urban and Social Change Review (Special Issue on Women and Work), I I, 28-36.

Rahkonen, O. and Takala, P. 1998. Social class differences in health and in functional disability among older men and women. International Journal of Health Services, 28, 3, $5^{\mathrm{II}-24}$.

Rogers, R. G. I996. The effects of family composition, health, and social support linkages on mortality. Fournal of Health and Social Behavior, 37, 4, 326-38.

Ross, C. E. and Mirowsky, J. I995. Does employment affect health? Fournal of Health and Social Behavior, 36, 3, 230-243.

Sarwari, A., Fredman, L., Langenberg, P. and Magaziner, J. 1998. Prospective study on the relation between living arrangements and change in functional health status of elderly women. American Fournal of Epidemiology, 147, 4, 370-8.

Schulz, R. and Beach, S. R. I999. Caregiving as a risk factor for mortality: the Caregiver Health Effects Study. Fournal of the American Fournal Association, 282, 23, 2215-I9.

Shye, D., Mullooly, J. P., Freeborn, D. K. and Pope, C. I995. Gender differences in the relationship between social network support and mortality: a longitudinal study. Social Science and Medicine, 41, 7, 935-47.

Sonnenberg, C., Beekman, A., Deeg, D. and van Tilburg, W. 200o. Sex differences in latelife depression. Acta Psychiatrica Scandinavica, ror , 4, 286-92.

Stansfeld, S. I999. Social support and social cohesion. In Marmot, M and Wilkinson, R. G. (eds), Social Determinants of Health. Oxford University Press, Oxford, I55-78.

Taylor, M. G. and Lynch, S. M. 2004. Trajectories of impairment, social support, and depressive symptoms in later life. Fournal of Gerontology, 59, 4, $\mathrm{S}_{238}{ }^{-4} 4$.

Thorslund, M. and Lundberg, O. I994. Health and inequalities among the oldest old. Journal of Aging \& Health, 6, I, 5 $\mathrm{I}^{-69}$.

Turner, R. J. and Noh, S. I988. Physical disability and depression: a longitudinal analysis. Fournal of Health and Social Behavior, 29, I, 23-37.

United Nations Organisation 2005. Living Arrangements of Older Persons Around the World. Department of Economic and Social Affairs, UNO, New York.

United Nations Organisation 2006. World Population Prospects: The 2006 Revision. UNO, New York. Available online at http://www.un.org/esa/population/publications/wpp20o6/ wpp20o6.htm. [Accessed February 2008].

Victoria, C. G., Huttly, S. R., Fuchs, S. C. and Olinto, M. T. 1997. The role of conceptual frameworks in epidemiological analysis: a hierarchical approach. International fournal of Epidemiology, 26, I, 224-7.

Von Dem Knesebeck, O., Wahrendorf, M., Hyde, M. and Siegrist, J. 2007. Socioeconomic position and quality of life among older people in io European countries: results of the SHARE study. Ageing \& Society, 27, 2, 269-84.

Vvorisalmi, M., Lintonen, T. and Jylhä, M. 2006. Comparative vs. global self-rated health: associations with age and functional ability. Aging Clinical and Experimental Research, I8, 3, 2 II -7 .

Walker, A., Pratt, C. and Eddy, L. I995. Informal caregiving to aging family members: a critical review. Family Relations, 44, 4, 402-II. 
Wethington, E. and Kessler, R. C. I986. Perceived support, received support, and adjustment to stressful life events. Fournal of Health and Social Behavior, 2, I, 78-89.

Zimmer, Z. 200I. A further discussion on revisiting the classification of household composition among elderly people. Fournal of Cross Cultural Gerontology, 18, 247-50.

Zunzunegui, M. V., Béland, F., Yacer, A. and León, V. 1998. Gender differences in depressive symptoms among Spanish elderly. Social Psychiatry and Psychiatric Epidemiology, 33, 5, $195^{-205}$.

Zunzunegui, M. V., Koné, A., Johri, M., Béland, F., Wolfson, C. and Bergman, H. 2004. Social networks and self-assessed health in two French-speaking Canadian community dwelling populations over 65. Social Science and Medicine, 58, I0, 2069-8I.

Address for correspondence:

Accepted 26 November 2008

Silvia Rueda Pozo, Universitat Pompeu Fabra,

G/ Ramón Trias Fargas, 25-27 o8005 Barcelona, Spain.

E-mail: silvia.rueda@upf.edu 